\title{
COVID-19 Pandemic: Impact on Health Care of Children and the Urgent Need to Restore Regular Healthcare Services
}

\author{
Rakesh Lodha $^{1}$ • S. K. Kabra ${ }^{1}$ \\ Received: 27 November 2020 / Accepted: 27 November 2020 / Published online: 8 January 2021 \\ (C) Dr. K C Chaudhuri Foundation 2021
}

The ongoing COVID-19 pandemic has affected all aspects of healthcare services. During the initial months, COVID-19 was thought of as a severe disease associated with high mortality and contagiousness. Anticipation of high disease load and high severity of illness created a fear among masses and also led policymakers to develop intensive care services and COVID-19 healthcare services aggressively. The emergency response to COVID-19 did come at the cost of care of other illnesses taking a back seat.

The emergency response to COVID-19 required reallocation of resources - both physical and human - to COVID care; these resources were drawn from the routine services. This reallocation has affected the out-patient, emergency, and in-patient services for non-COVID conditions. While new physical infrastructure could be deployed, the major constraint is trained manpower. At the same time, requirements for PPE to prevent infections among the healthcare professionals (HCPs) has affected the productivity.

Directly or indirectly, non-COVID illnesses had inadequate access to healthcare services, illnesses were missed, and there was break in the services for chronic illnesses. Routine preventive and promotive health services were also affected. A substantial effort will be required to fill up the gaps thus created, especially for childhood immunization. In a modeling analysis of impact of the COVID-19 pandemic on the tuberculosis (TB) epidemic in three high-burden countries, it was estimated that a 3-month suspension of TB services, followed by 10 months to restore to normal, may cause, over the next 5 years, an additional 1.19 million TB cases (credible interval 1.06-1.33) and 361,000 TB deaths (credible interval 333-394 thousand) in India; the major reason of this impact is the accumulation of undetected TB during lockdown [1].

S. K. Kabra

skkabra@live.in

1 Department of Pediatrics, All India Institute of Medical Sciences, New Delhi, India
A significant reduction in numbers seeking care in the emergency services for non-COVID illnesses are now being highlighted [2-5]. Some groups/societies, have started issuing recommendations for handling children with specific problems [6]; however, children with chronic illnesses suffered as non-COVID care was largely restricted to critically-ill children.

We have observed that COVID-19 has impacted services to children with chronic respiratory problems in our center. Annually, we register about 500 new patients with chronic respiratory problems in the Pediatric Pulmonology services; however, from March 2020 till November 2020, there were no new registrations of children with chronic respiratory problem. Prior to March 2020, we provided consultations in the specialty services to $130-140$ children with chronic respiratory problems every week. As physical OPDs were closed and subsequently, restricted OPDs were started, we provided teleconsultation to 60-70 per week, this implied a $>50 \%$ reduction during March to November 2020. The beneficiaries of these tele-consultations were mainly follow-up patients. Further, about 2-3 children were diagnosed with cystic fibrosis (CF) in a month; during March to November 2020, we did not diagnose a single case of $\mathrm{CF}$ as no routine OPD services were available. We had registered 180 new children with TB during March to October 2019, while during corresponding period in 2020 , only 5 children were diagnosed with TB, who got hospitalized through emergency room due to severe illness. These indicative observations suggest that these illnesses are missed and those in follow-up got less than optimal care. While telemedicine was better than no care, it is unlikely to be a complete replacement for physical visits and evaluations. Impact of missed or delayed diagnosis of conditions such as CF or TB is quite serious. Similarly, less optimal treatment to children with chronic respiratory problem can affect the final outcome, that we are likely to encounter in the coming months and years.

The above examples emphasize that diagnosis of many illnesses have been missed and children are expected to visit healthcare facilities in advanced stages of illness whenever 
normalcy of routine services is restored. Additionally, care of all chronic illnesses was suboptimal and will affect the outcome, that will be seen in coming years. Healthcare facilities should be ready to overcome these problems.

At the beginning of pandemic, the medical fraternity was unclear about various aspects of COVID-19. Now it is clear that this novel viral infection has significant secondary attack rate; in children, majority of the disease is mild or moderate severity with favorable outcome with supportive care. Overall case fatality is low except in those with underlying illness [7-9]. It is also clear that the COVID-19 will not disappear completely even if we have effective vaccine; the virus is likely to remain in circulation for a few years. It is important to realize that the pandemic has adversely affected care of all other illnesses and there is a need to restore routine services.

The challenges for restoration of non-COVID services include scaling back the emergency COVID-19 response, which is dependent of the state of the pandemic; having adequate manpower to cover both COVID- and non-COVID services. The pandemic has strained the healthcare services in countries with robust healthcare systems; this is even more evident in countries where the health systems, especially, the public health systems are weak. Even in the pre-COVID period, the capacity of the health systems to provide adequate care in these countries was limited; with the pandemic, the strain on the capacity is even greater.

For effective restoration of non-COVID services, there is need for reopening of services in a phased manner with creating adequate infrastructure, confidence building among HCPs, preparing to handle periodic surge in infection, developing strategies for control measures at community level and preparing for next few years. Confidence building measures among HCPs include sharing data about protective efficacy of masks and general measures, ensuring access to optimal personal protective equipment, of which respiratory protection is the main one, and emphasizing proper use even when the vaccine is available. The capacity for COVID-19 testing needs a major scale-up, to provide unrestricted access to COVID-19 testing; this will increase the confidence among HCPs. More accurate point-of-care tests is the need of the hour. The surveillance for COVID-19 should continue; possible events that could indicate an upsurge in caseloads should be identified and the healthcare systems should be prepared to handle these situations appropriately.

The need for the emergency response to COVID-19 pandemic is likely to continue for many months. The high secondary attack rates, high likelihood of patients, and HCPs getting COVID-19 in healthcare settings will continue to warrant separate facilities for COVID-19 care till specific treatments are available. Even though in the large majority of the population, the illness is mild, there is still need for all to adhere to the guidelines for prevention of COVID-19. Till an effective vaccine is available, adherence to universal masking, hand hygiene, and social distancing are the keys to prevention. Additional efforts need to be in place to protect the high-risk groups.

A phased opening of non-COVID services without creating apprehensions among general public as well as HCPs or increasing the risk of surge in infections is the need of the hour and should be initiated as soon as possible. Facility-specific plans should be developed to ensure protection of both patients and HCPs from exposure to the virus, while accounting for shortage of HCPs and infrastructure (both for the care of patients with COVID-19 and non-COVID conditions), and prioritizing the access to patients with various acute and chronic conditions. Optimal screening, adequate facilities for diagnostic testing, ensuring physical distancing, adherence to infection prevention and control practices, are essential components to facilitate restoration of services.

\section{Compliance with Ethical Standards}

Conflict of Interest None.

\section{References}

1. Cilloni L, Fu H, Vesga JF, et al. The potential impact of the COVID19 pandemic on the tuberculosis epidemic a modelling analysis. EClinicalMedicine. 2020;28:100603.

2. Dopfer C, Wetzke M, Zychlinsky Scharff A, et al. COVID-19 related reduction in pediatric emergency healthcare utilization - a concerning trend. BMC Pediatr. 2020;20:427.

3. Nicholas DB, Belletrutti M, Dimitropoulos G, et al. Perceived impacts of the COVID-19 pandemic on pediatric care in Canada: a roundtable discussion. Glob Pediatr Health. 2020;7: $2333794 X 20957652$.

4. Valitutti F, Zenzeri L, Mauro A, et al. Effect of population lockdown on pediatric emergency room demands in the era of COVID-19. Front Pediatr. 2020;8:521.

5. Raman R, Madhusudan M. Impact of the COVID-19 pandemic on admissions to the pediatric emergency department in a tertiary care hospital. Indian J Pediatr. 2020. https://doi.org/10.1007/s12098-02003562-y.

6. Garg V, Bakhshi S, Gupta G, Pushpam D. Managing pediatric cancer patients in COVID19 pandemic. Indian J Pediatr. 2020;87:558.

7. Swann OV, Holden KA, Turtle L, et al; ISARIC4C Investigators. Clinical characteristics of children and young people admitted to hospital with covid-19 in United Kingdom: prospective multicentre observational cohort study. BMJ. 2020;370:m3249.

8. van der Zalm MM, Lishman J, Verhagen LM, et al. Clinical experience with SARS CoV-2 related illness in children - hospital experience in Cape Town, South Africa. Clin Infect Dis. 2020;ciaa1666. https:// doi.org/10.1093/cid/ciaa1666.

9. Nallasamy K, Angurana SK, Jayashree M, et al; for Pediatric COVID management team. Clinical profile, hospital course and outcome of children with COVID-19. Indian J Pediatr. 2020. https://doi. org/10.1007/s12098-020-03572-w.

Publisher's Note Springer Nature remains neutral with regard to jurisdictional claims in published maps and institutional affiliations. 